\title{
Optical klystron and harmonic generation free electron laser
}

\author{
Qika Jia \\ National Synchrotron Radiation Laboratory, University of Science and Technology of China, Hefei, Anhui, 230029, China
} (Received 24 September 2004; published 20 June 2005)

\begin{abstract}
The optical field evolution of an optical klystron free electron laser is analytically described for both low gain and high gain cases. The harmonic optical klystron (HOK) in which the second undulator is resonant on the higher harmonic of the first undulator is analyzed as a harmonic amplifier. The optical field evolution equation of the HOK is derived analytically for both the CHG mode (coherent harmonic generation, the quadratic gain regime) and the HGHG mode (high gain harmonic generation, the exponential gain regime), the effects of energy spread, energy modulation, and dispersion in the whole process are taken into account. The linear theory is given and discussed for the HGHG mode. The analytical formula is given for the CHG mode.
\end{abstract}

DOI: 10.1103/PhysRevSTAB.8.060701

PACS numbers: 41.60.Cr, 42.25.Kb, 42.65.Ky

\section{INTRODUCTION}

An optical klystron $(\mathrm{OK})$ consists of two undulators separated by a dispersive section. In the first undulator (modulation section) the energy modulation of the electron beam is induced by interaction with the optical field. It is transformed into density modulation (bunching) after passing the dispersive section, then the radiation in the second undulator (gain section) is coherently enhanced [1]. An optical klystron gives a higher small signal gain than an undulator. In order to reduce the total length of the undulator for SASE free-electron laser (FEL), an optical klystron operating in high gain regime has been proposed and discussed [2,3]. Optical klystron also has been used for coherent harmonic generation $(\mathrm{CHG})[4,5]$. In this scheme an external laser pulse is focused into the first undulator, the wavelength of the laser is resonant with the fundamental radiation of the optical klystron, with optimized system parameters the harmonic radiation in the second undulator is coherently enhanced. Analysis has shown that if the second undulator of an OK is resonant on a higher harmonic, namely, the wavelength of fundamental radiation of the second undulator matches with $n$th harmonic optical field in the first undulator, it will enhance the harmonic generation [6]. To distinguish it from the normal optical klystron, we temporarily call such optical klystron the "harmonic optical klystron" (HOK). A similar configuration was proposed and used for high gain harmonic generation (HGHG) [7], the scheme evolved from many earlier ideals (e.g., Ref. [8]). In the HGHG mode the optical power grows exponentially while in the CHG mode the optical power grows quadratically. Cascaded optical klystron [9,10] and cascaded harmonic optical klystron [11-13] for x-ray FEL are also proposed and discussed.

The theory of optical klystron (and harmonic optical klystron) has been studied by many authors (e.g., Refs. [1-4,14-16]) . The small signal gain of optical

*Electronic address: jiaqk@ustc.edu.cn klystron is given by using Madey's theorem via the derivation of spontaneous spectrum [14], or by using the coupled Lorentz-Maxwell equations [15] but in a rather lengthy form. In Ref. [7] the HGHG problem is solved for the small energy-spread limit, in the second undulator the electron beam is assumed to be monoenergetic and dispersive effect is ignored. The amplifying process of optical klystron (and harmonic optical klystron) has been analyzed mostly by calculating the bunching factor at the entrance of the second undulator (the techniques developed for microwave klystron). In this paper I derive the optical field evolution equation completely analytically for $\mathrm{OK}$ and HOK. The energy-spread effect and the dispersive effect in the whole process will be considered in the derivation.

\section{OPTICAL KLYSTRON}

We use the one-dimensional FEL theory and start from the paraxial optical field equation and the electron phase equation:

$$
\begin{gathered}
\frac{d \tilde{a}_{s}}{d z}=\lambda_{s} r_{e} a_{u} \delta_{p} n_{e}\left\langle\frac{e^{-i \phi}}{\gamma}\right\rangle, \\
\frac{d^{2} \phi}{d z^{2}}=-\frac{2 k_{u} k_{s} a_{u} \delta_{p}}{\gamma^{2}} \operatorname{Re}\left(\tilde{a}_{s} e^{i \phi}\right),
\end{gathered}
$$

where $\tilde{a}_{s}=a_{s} e^{i \varphi_{s}}, \quad a_{s}=e E_{s} /\left(m c^{2} k_{s}\right) \quad$ and $a_{u}=$ $e B_{u} /\left(m c^{2} k_{u}\right)$ are the dimensionless vector potential of the rms radiation field $E_{s}$ and undulator field $B_{u}$, respectively; $k_{s}=2 \pi / \lambda_{s}, k_{u}=2 \pi / \lambda_{u}$ are the corresponding wave number; $\varphi_{s}$ is the phase of radiation field; $\phi=\left(k_{s}+\right.$ $\left.k_{u}\right) z-\omega_{s} t$ is the ponderomotive phase of electron, $r_{e}$ is the classical electron radius; $n_{e}$ and $\gamma$ is the density and energy of electrons; the angular bracket represents the average over the electron's initial phases and initial phase velocities. $\delta_{p}$ is the polarization modify factor: for circularly polarized helical undulator $\delta_{p}=1$; for linearly polarized planar undulator with even $n$th harmonic radiation $\delta_{p}=0$, 
and with odd $n$th harmonic $\delta_{p}=[J, J]_{n}$,

$$
\begin{aligned}
{[J, J]_{n}=} & (-1)^{(n-1) / 2}\left[J_{(n-1) / 2}\left(\frac{n a_{u}^{2}}{2\left(1+a_{u}^{2}\right)}\right)\right. \\
& \left.-J_{(n+1) / 2}\left(\frac{n a_{u}^{2}}{2\left(1+a_{u}^{2}\right)}\right)\right]
\end{aligned}
$$

$J$ is integer order Bessel function. We consider an optical klystron where the two undulator sections have the same magnetic field parameter $a_{u 1}=a_{u 2}, k_{u 1}=k_{u 2}$ but different length.

In the second undulator the electron phase is

$$
\phi_{2}=\phi_{20}+\phi_{20}{ }^{\prime} z_{2}+\Delta \phi_{2}
$$

The first term of the right hand side of Eq. (3) is the electron phase at the entrance of the second undulator

$$
\phi_{20}=\phi_{1}\left(l_{1}\right)+\Delta \phi_{d}=\phi_{10}+\phi_{10}{ }^{\prime} l_{1}+\Delta \phi_{1}+\Delta \phi_{d},
$$

where $\phi_{10}$ and $\phi_{10}{ }^{\prime}$ is the initial phase and phase velocity (detuning parameter), $\Delta \phi_{1}$ is the phase change due to interaction with radiation field in the first undulator and given from Eq. (2),

$$
\begin{aligned}
\Delta \phi_{1} & =-2 k_{s} k_{u} a_{u} \delta_{p} \operatorname{Re} \iint \frac{\tilde{a}_{s} e^{i \phi}}{\gamma^{2}} d z^{\prime \prime} d z^{\prime} \\
& =-2 k_{s} k_{u} a_{u} \delta_{p} \operatorname{Re} \int_{0}^{l_{1}}\left(l_{1}-z_{1}\right) \frac{\tilde{a}_{s} e^{i \phi_{1}}}{\gamma^{2}} d z_{1} .
\end{aligned}
$$

$\Delta \phi_{d}$ is phase change in the dispersive section

$$
\begin{gathered}
\Delta \phi_{d}=\int_{0}^{d} k_{s}\left(1-\frac{1}{\beta_{\mathrm{II}}}\right) d z=-\frac{\gamma_{r}^{2}}{\gamma^{2}} 2 \pi N_{d} \\
=N_{d} \lambda_{u} \phi_{1}{ }^{\prime}\left(l_{1}\right)-2 \pi N_{d}, \\
N_{d}=\frac{1}{\lambda_{u}\left(1+a_{u}^{2}\right)}\left[d+\left(\frac{e}{m c^{2}}\right)^{2} \int_{0}^{d}\left(\int_{0}^{z} B_{d} d z^{\prime}\right)^{2} d z\right]
\end{gathered}
$$

where $d$ and $B_{d}$ are the lengths and magnetic field of the dispersive section, respectively, $\gamma_{r}$ is the resonant energy; the resonant relation of FEL and the phase velocity expression $\phi^{\prime}=k_{u}\left[1-\left(\gamma_{r}^{2} / \gamma^{2}\right)\right]$ is used. By its definition expression [Eq. (7)] the dispersive section parameter $N_{d}$ is the scale parameter of optical klystron itself and independent on the electron beam.

The second term of Eq. (3) $\phi_{20}^{\prime}$ is electron phase velocity at the entrance of the second undulator

$$
\begin{gathered}
\phi_{20}{ }^{\prime}=\phi_{1}{ }^{\prime}\left(l_{1}\right)=\phi_{10}{ }^{\prime}+\Delta \phi_{1}{ }^{\prime}, \\
\Delta \phi_{1}{ }^{\prime}=-2 k_{s} k_{u} a_{u} \delta_{p} \operatorname{Re} \int_{0}^{l_{1}} \frac{\tilde{a}_{s} e^{i \phi_{1}}}{\gamma^{2}} d z_{1}
\end{gathered}
$$

The third term of Eq. (3) $\Delta \phi_{2}$ is phase variation due to the interaction with the radiation field in the second undulator [same as Eq. (5), but $l_{1} \rightarrow z_{2}, z_{1} \rightarrow z_{2}{ }^{\prime}, \phi_{1} \rightarrow \phi_{2}$ ].
Therefore Eq. (3) becomes

$$
\begin{aligned}
\phi_{2}= & \phi_{10}-2 \pi N_{d}+\phi_{10}{ }^{\prime}\left(l_{1}+N_{d} \lambda_{u}+z_{2}\right) \\
& -2 k_{s} k_{u} a_{u} \delta_{p} \operatorname{Re} \int_{0}^{l_{1}}\left(l_{1}-z_{1}+N_{d} \lambda_{u}+z_{2}\right) \\
& \times \frac{\tilde{a}_{s} e^{i \phi_{1}}}{\gamma^{2}} d z_{1}+\Delta \phi_{2} .
\end{aligned}
$$

Substituting the above expression into Eq. (1) we yield the optical field evolution equation for linear regime in the second undulator

$$
\begin{aligned}
\frac{d \tilde{a}_{s}}{d z_{2}}= & -\left(2 k_{u} \rho\right)^{3}\left\langle\frac { \partial } { \partial \phi _ { 1 0 } { } ^ { \prime } } \left[ e^{-i\left[\phi_{10}{ }^{\prime}\left(l_{1}+N_{d} \lambda_{u}\right)-2 \pi N_{d}\right]}\right.\right. \\
& \times \int_{0}^{l_{1}} \tilde{a}_{s} e^{-i \phi_{10}{ }^{\prime}\left(z_{2}-z_{1}\right)} d z_{1} \\
& \left.\left.+\int_{0}^{z_{2}} \tilde{a}_{s} e^{-i \phi_{10}{ }^{\prime}\left(z_{2}-z_{2}{ }^{\prime}\right)} d z_{2}{ }^{\prime}\right]\right\rangle_{\phi_{10}{ }^{\prime}}
\end{aligned}
$$

where $\rho$ is the Pierce parameter $\rho=(1 / 2 \gamma) \times$ $\left\{\left(r_{e} n_{e} \lambda_{u}^{2} a_{u}^{2} \delta_{p}^{2}\right) / 2 \pi\right\}^{1 / 3}$. When $N_{d}=0$ the above equation recovers the optical field equation for the normal undulator. Multiplying two sides of Eq. (10) by $\tilde{a}_{s}^{*}$ and taking real parts, then using the simplification method in Ref. [17], we obtain the small signal gain of optical klystron

$$
\begin{aligned}
g= & \frac{-\left(2 k_{u} \rho\right)^{3}}{\left|a_{s 0}\right|^{2}}\left\langle\frac{\partial}{\partial \phi_{10}{ }^{\prime}}\right| \int_{0}^{l_{1}} \tilde{a}_{s} e^{i \phi_{10}{ }^{\prime} z} d z \\
& \left.+\left.e^{i\left[\phi_{10}{ }^{\prime}\left(l_{1}+N_{d} \lambda_{u 1}\right)-2 \pi N_{d}\right]} \int_{0}^{l_{2}} \tilde{a}_{s} e^{i \phi_{10}{ }^{\prime} z} d z\right|^{2}\right\rangle_{\phi_{10}{ }^{\prime}}
\end{aligned}
$$

Actually this is the result given in Ref. [18] if $N_{d}$ is an integer. For low gain case $\tilde{a}_{s} \approx \tilde{a}_{s 0}$ in the integral of the right hand side of Eq. (11), and for large $N_{d}$ it can be proved that the gain would be maximum when optical klystron is symmetric configuration $\left(l_{1}=l_{2}=l\right)$ [6]. Then the small signal gain becomes

$$
\begin{aligned}
g= & -\left(2 k_{u} \rho\right)^{3} 2 l^{2}\left\langle\frac { \partial } { \partial \phi _ { 1 0 } { } ^ { \prime } } \left\{\left[ 1+\cos \left(\phi_{10}{ }^{\prime}\left(l+N_{d} \lambda_{u}\right)\right.\right.\right.\right. \\
& \left.\left.\left.\left.-2 \pi N_{d}\right)\right] \sin c^{2}\left(\frac{\phi_{10}{ }^{\prime} l}{2}\right)\right\}\right\rangle_{\phi_{10}{ }^{\prime}} \\
\approx & (8 \pi N \rho)^{3} \frac{1}{4}\left(1+\frac{N_{d}}{N}\right)\left\langle\sin \left[\phi_{10}{ }^{\prime}\left(N+N_{d}\right) \lambda_{u}-2 \pi N_{d}\right]\right. \\
& \left.\times \sin ^{2}\left(\frac{\phi_{10}{ }^{\prime} l}{2}\right)\right\rangle_{\phi_{10}{ }^{\prime}}
\end{aligned}
$$

where the approximation is made for a large $N_{d}$. From Eq. (12) we can see that the fractional part of $N_{d}$ causes a phase shift for gain curve, $2 \pi N_{d}$ is the $\delta \psi$ defined in Ref. [19].

For the high gain case, here we only indicate that Eq. (10) is also valid. 


\section{HARMONIC OPTICAL KLYSTRON WITH SEEDING LASER}

For the HOK generally the wavelength of the $n_{1}$ th harmonic radiation field in the first undulator matches with the $n_{2}$ th harmonic radiation field of the second undulator, namely, one has

$$
\lambda_{u 2}\left(1+a_{u 2}^{2}\right) / n_{2}=\lambda_{u 1}\left(1+a_{u 1}^{2}\right) / n_{1} .
$$

In the following we consider the case of $n_{1}=n, n_{2}=1$. Now the initial electron phase in the second undulator is

$$
\phi_{20}=n \phi_{1}\left(z_{20}\right)+\left(k_{u 2}-n k_{u 1}\right) z_{20},
$$

$\phi_{1}\left(z_{20}\right)$ is given by Eq. (4). The second part in the right hand side of Eq. (14) is a constant for all electrons. The initial electron phase velocity in the second undulator is

$$
\phi_{20}{ }^{\prime}=k_{u 2}\left(1-\frac{\gamma_{r}^{2}}{\gamma_{20}^{2}}\right)=\frac{k_{u 2}}{k_{u 1}} \phi_{1}{ }^{\prime}\left(l_{1}\right) .
$$

Thus the electron phase in the second undulator is (we drop the constant term)

$$
\begin{aligned}
\phi_{2}= & n \phi_{10}+\phi_{10}{ }^{\prime}\left[n\left(l_{1}+N_{d} \lambda_{u 1}\right)+z_{2} \lambda_{u 1} / \lambda_{u 2}\right] \\
& -\frac{2 k_{u 1} k_{s 1} a_{u 1} \delta_{p 1}}{\gamma^{2}} \operatorname{Re} \int_{0}^{l_{1}}\left[n\left(l_{1}-z_{1}+N_{d} \lambda_{u 1}\right)\right. \\
& \left.+z_{2} \lambda_{u 1} / \lambda_{u 2}\right] \tilde{a}_{s 1} e^{i \phi_{1}} d z_{1}+\Delta \phi_{2},
\end{aligned}
$$

where $k_{s 1}$ and $a_{s 1}$ are the wave number and the dimensionless vector potential of the seed laser field (rms), respectively. The harmonic generation problem of $\mathrm{HOK}$ including the electron beam quality effects and dispersive effects for the whole process from beginning to saturation can be numerically solved by substituting Eq. (16) into Eq. (1).

For harmonic amplifier FEL the seed laser field is not weak, so in the right hand side of Eq. (16) the third term (the phase variation due to the interaction with the seeding optical field) cannot be treated as a small quantity to expand, according to the procedure exploited in obtaining Eq. (10). Owing to the short length of the first section undulator (modulator) the optical field in the modulator can be considered approximately constant. The integral in this term varies approximately linearly with $z_{1}$, so taking its median in the integral is a reasonable approximation

$$
\begin{aligned}
- & \frac{2 k_{u 1} k_{s 1} a_{s 1} a_{u 1} \delta_{p 1}}{\gamma^{2}} \int_{0}^{l_{1}}\left[n\left(l_{1}-z_{1}+N_{d} \lambda_{u 1}\right)\right. \\
& \left.+z_{2} \lambda_{u 1} / \lambda_{u 2}\right] \cos \left(\phi_{10}+\phi_{10}{ }^{\prime} z_{1}\right) d z \\
& \approx-n \Delta \xi \cos \left(\phi_{10}+\phi_{10}{ }^{\prime} \frac{l_{1}}{2}\right)
\end{aligned}
$$

where

$$
\begin{aligned}
\Delta \xi & =\frac{4 \pi k_{s 1} a_{s 1} a_{u 1} \delta_{p 1}}{\gamma^{2}}\left(\frac{N_{1}}{2}+N_{d}+\frac{z_{2}}{n \lambda_{u 2}}\right) l_{1} \\
& =4 \pi\left(\frac{N_{1}}{2}+N_{d}+\frac{z_{2}}{n \lambda_{u 2}}\right) \frac{\Delta \gamma_{m}}{\gamma}
\end{aligned}
$$

$\Delta \gamma_{m} / \gamma$ is the maximum energy modulation induced in the first section undulator

$$
\frac{\Delta \gamma_{m}}{\gamma}=4 \pi N_{1} \frac{a_{u 1} \delta_{p 1} a_{s 1}}{\left(1+a_{u 1}^{2}\right)} .
$$

Substituting Eqs. (16) and (17) into Eq. (1) and linearizing it

$$
\begin{aligned}
e^{-i \phi_{2} \approx} & e^{-i\left\{n\left[\phi_{10}+\phi_{10}{ }^{\prime}\left(l_{1}+N_{d} \lambda_{u 1}\right)-\Delta \xi \cos \left(\phi_{10}+\phi_{10}{ }^{\prime} l_{1} / 2\right)\right]+\phi_{02}{ }^{\prime} z_{2}\right\}}\left(1-i \Delta \phi_{2}\right) \\
= & f\left(\phi_{10}\right)+e^{-i\left\{n \phi_{10}{ }^{\prime}\left[\left(l_{1} / 2\right)+N_{d} \lambda_{u 1}\right]+\phi_{02}{ }^{\prime} z_{2}\right\}} i^{n} J_{n}(n \Delta \xi)-k_{s 2} k_{u 2} a_{u 2} \delta_{p 2} \frac{\partial}{\partial \phi_{02}{ }^{\prime}} \int_{0}^{z_{2}} J_{0}\left[2 k_{u 2}\left(z_{2}-z_{2}{ }^{\prime}\right) \frac{\Delta \gamma_{m}}{\gamma}\right] \\
& \times \frac{\tilde{a}_{s 2}}{\gamma^{2}} e^{-i \phi_{02}{ }^{\prime}\left(z_{2}-z_{2}{ }^{\prime}\right)} d z_{2}{ }^{\prime}+i k_{s 2} k_{u 2} a_{u 2} \delta_{p 2} e^{-i 2 n \phi_{10}{ }^{\prime}\left[\left(l_{1} / 2\right)+N_{d} \lambda_{u 1}\right]} \int_{0}^{z_{2}}\left(z_{2}-z_{2}{ }^{\prime}\right) J_{2 n}[n(\Delta \xi+\Delta \xi)] \\
& \times \frac{\tilde{a}_{s 2}}{\gamma^{2}} e^{-i \phi_{02}{ }^{\prime}\left(z_{2}+z_{2}{ }^{\prime}\right)} d z_{2}{ }^{\prime} .
\end{aligned}
$$

Here $f\left(\phi_{10}\right)$ is all other terms that depend on $\phi_{10}$. It will be null after averaging over a uniform initial phase distribution of electrons; we denote $\left(\lambda_{u 1} / \lambda_{u 2}\right) \phi_{10}{ }^{\prime}$ as $\phi_{02}{ }^{\prime}$ (note it is different with $\phi_{20}{ }^{\prime}$ ), it is the electron phase velocities (referenced to the second undulator) at the entrance of the first undulator:

$$
\phi_{02}^{\prime}=\frac{k_{u 2}}{k_{u 1}} \phi_{10}^{\prime}=k_{u 2}\left(1-\frac{\gamma_{r}^{2}}{\gamma_{0}^{2}}\right)
$$

Finally the optical field evolution equation in the linear region for HOK is given by 


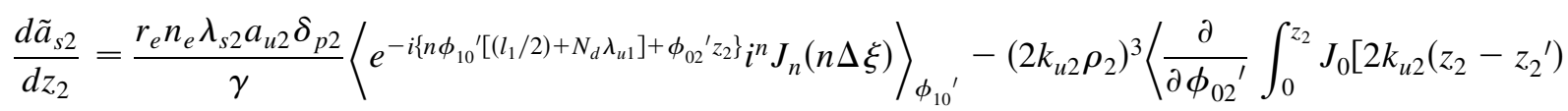

$$
\begin{aligned}
& \left.\left.\times \frac{\Delta \gamma_{m}}{\gamma}\right] \tilde{a}_{s 2} e^{-i \phi_{02}{ }^{\prime}\left(z_{2}-z_{2}{ }^{\prime}\right)} d z_{2}{ }^{\prime}\right\rangle_{\phi_{10}{ }^{\prime}}+\left(2 k_{u 2} \rho_{2}\right)^{3}\left\langle i e^{-i 2 n \phi_{10}{ }^{\prime}\left[\left(l_{1} / 2\right)+N_{d} \lambda_{u 1}\right]} \int_{0}^{z_{2}}\left(z_{2}-z_{2}{ }^{\prime}\right) J_{2 n}[n(\Delta \xi\right. \\
& \left.\left.\left.+\Delta \xi^{\prime}\right)\right] \tilde{a}_{s 2} e^{-i \phi_{02}{ }^{\prime}\left(z_{2}+z_{2}{ }^{\prime}\right)} d z_{2}{ }^{\prime}\right\rangle_{\phi_{10}{ }^{\prime}}
\end{aligned}
$$

In the right hand side of the above equation, the first term corresponds to the coherent enhancement process. We can see that the dispersion effect $(\Delta \xi)$ and energy-spread effect (the exponential factor) include the contribution not only from the dispersive section, the modulation section, but also from the gain section. The second term corresponds to the usual gain process. It gives usual gain results when the seed laser is off. The Bessel function in it indicates the effect of the additional energy spread due to energy modulation. The contribution of the third term is small and can be neglected in many cases.

Figure 1 is a numerical result of Eq. (22) compared with the result given by solving Eqs. (1) and (16) (for $5.3 \mu \mathrm{m}$ HGHG experiment parameters of Ref. [20]). It shows that the linear approximation is valid from the start up to near saturation (linear region). For a harmonic optical klystron with seeding laser the amplifying process has two parts: the coherent enhancement process and the usual gain process. For the initial distance $z_{2}$ of the radiator the usual gain process is in the low gain regime, the coherent enhancement process is dominant; this is the CHG mode. As the radiator distance $z_{2}$ increases the usual gain process enters the high gain regime (the exponential gain regime) and becomes dominant; this is the HGHG mode.

For the HGHG mode, the electron beam current is relatively high, and the length of the second undulator must be sufficiently long to reach the exponential gain regime. The asymptotic approximate solution can be given

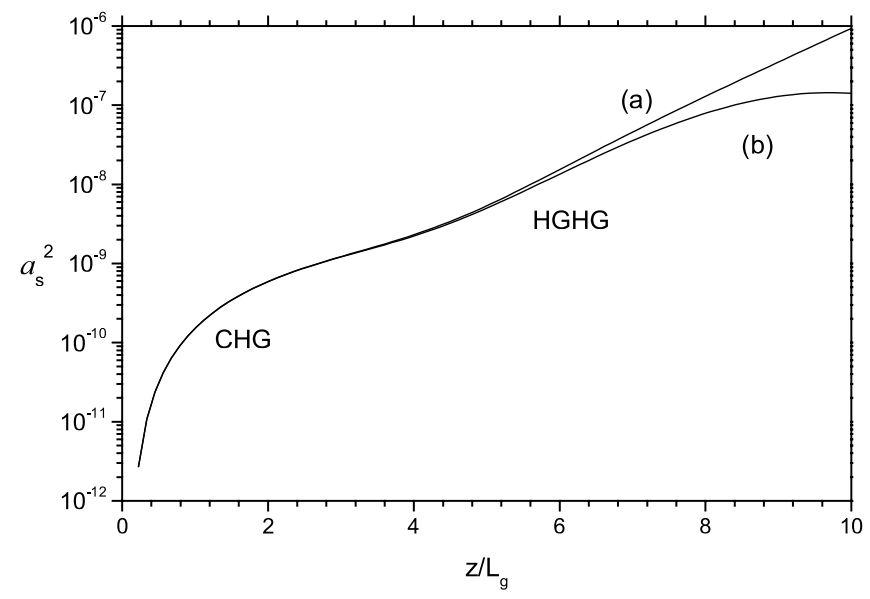

FIG. 1. (a) The linear approximation [Eq. (22)] compared with (b) the result given by numerically solving Eqs. (1) and (16). for monoenergetic electron beam and weak modulation [21]

$$
a_{s 2}\left(z_{2}\right) \approx \frac{4 k_{u 2} \gamma^{2} \rho_{2}^{2}}{3 k_{s 2} a_{u 2} \delta_{p 2}} J_{n}\left(n \Delta \xi^{*}\right) e^{\sqrt{3} k_{u 2} \rho_{2} z_{2}},
$$

where $\Delta \xi^{*}=\Delta \xi\left(z_{2}{ }^{*}\right), z_{2}{ }^{*}: 0<z_{2}{ }^{*}<l_{2}$

$$
\begin{aligned}
\int_{0}^{l_{2}} J_{n}\left[n \Delta \xi\left(z_{2}\right)\right] e^{-i \phi_{02}{ }^{\prime} z_{2}} d z_{2}= & J_{n}\left[n \Delta \xi\left(z_{2}{ }^{*}\right)\right] \\
& \times \int_{0}^{l_{2}} e^{-i \phi_{02}{ }^{\prime} z_{2}} d z_{2} .
\end{aligned}
$$

In the CHG mode, the electron beam current is low and the length of the radiator section of HOK is short. We can only consider the coherent enhancement process [the first term of Eq. (22)]. Because $N_{2}$ is small while the $N_{d}$ may be very large, it has $N_{d}+N_{1} / 2 \gg N_{2} / n \geq z_{2} / n \lambda_{u 2}$ (for example, in our CHG project $N_{1}=10, N_{2}=12, N_{d} \cong 90$ [22]). Therefore the $z_{2}$ in $\Delta \xi$ [Eq. (18)] can be approximated with its median in the second undulator. Thus we give the analytical result for the optical field of HOK in the CHG mode

$$
\begin{aligned}
\tilde{a}_{s 2}= & -\frac{r_{e} \lambda_{s 2} a_{u 2} \delta_{p 2} n_{e}}{\gamma} J_{n}(n \Delta \xi) \\
& \times\left\langle i^{n} e^{-i \phi_{10}{ }^{\prime} \lambda_{u 1}\left\{n\left[\left(N_{1} / 2\right)+N_{d}\right]+\left(N_{2} / 2\right)\right\}} l_{2} \sin c \frac{\phi_{02}{ }^{\prime} l_{2}}{2}\right\rangle_{\phi_{10}{ }^{\prime}} .
\end{aligned}
$$

For a Gaussian initial energy distribution of the electron beam the corresponding radiation intensity is

$$
\begin{aligned}
\tilde{a}_{s 2}^{2} & =\left(\frac{r_{e} \lambda_{s 2} a_{u 2} \delta_{p 2} n_{e} l_{2}}{\gamma}\right)^{2} J_{n}^{2}(n \Delta \xi) f_{\gamma}^{2}, \\
f_{\gamma} & =\exp \left\{-\frac{1}{2}\left[4 \pi n\left(N_{d}+\frac{N_{1}+N_{2} / n}{2}\right) \frac{\sigma_{\gamma}}{\gamma}\right]^{2}\right\} .
\end{aligned}
$$

If we substitute in Eq. (25)

$$
a_{u 2} \rightarrow a_{u}, \quad \delta_{p 2}=[J, J]_{1} \rightarrow \delta_{p}=[J, J]_{n}, \quad N_{2} / n \rightarrow N_{2},
$$

then we have the $n$th harmonic radiation intensity for OK configuration. The advantage of $\mathrm{HOK}$ over $\mathrm{OK}$ for $\mathrm{CHG}$ is obvious: the energy spread effect is reduced, the radiation is also enhanced by proper selecting undulator parameters to make $\left(\left(a_{u 2}[J, J]_{1}\right)^{2} \gg\left(a_{u}[J, J]_{n}\right)^{2}[6]\right.$. Moreover besides the odd harmonic the HOK also can be operated at the even harmonic of the seed laser. 
From Eqs. (23) and (25) the division of the CHG mode (quadratic gain regime) and the HGHG mode (the exponential gain regime) for monoenergetic electron beam can be estimated: $z_{2} \approx 3.73 L_{g}\left[L_{g}=1 /\left(2 \sqrt{3} k_{u} \rho\right)\right]$ namely about four gain length. Figure 2 gives a comparison of the analytical formula [Eq. (25)] and the linear theory [Eq. (22)] for the CHG mode (using the parameters of Ref. [22]). It can be seen that the agreement between them is very good.

For the CHG mode when modulation is weak it has $J_{n}(n \Delta \xi) \cong(n \Delta \xi)^{n}$ in Eq. (25), then the optimal dispersive parameter can be given as

$$
N_{d}^{\mathrm{opt}}=\frac{1}{4 \pi \sqrt{n} \sigma_{\gamma} / \gamma}-\frac{N_{1}+N_{2} / n}{2}
$$

When the energy spread is small, the dispersive field and the seeding laser field should be chosen to make $J_{n} \cong$ $J_{n, \text { max }}$, in this instance it has $n \Delta \xi \cong n+1$

$$
N_{d}^{\mathrm{opt}}=\frac{n+1}{4 \pi n \Delta \gamma_{m} / \gamma}-\frac{N_{1}+N_{2} / n}{2}
$$

From Eq. (22) we noted that for the linear region the additional energy spread due to energy modulation only affects usual gain term but not the coherent enhancement term. Therefore, for the CHG scheme, in which the coherent enhancement term is dominant, generally one should chose a large seeding laser field $a_{s 1}$ (strong modulation) and a small $N_{d}$ (weak dispersion, to reduce the effect of energy spread) to make $J_{n} \cong J_{n \text {, max }}$.

For the HGHG scheme the additional energy-spread effect due to energy modulation must be considered, the optimization of dispersive parameter and the seeding laser field is more complicated [23]. The additional energyspread restriction gives the up limit for seeding laser

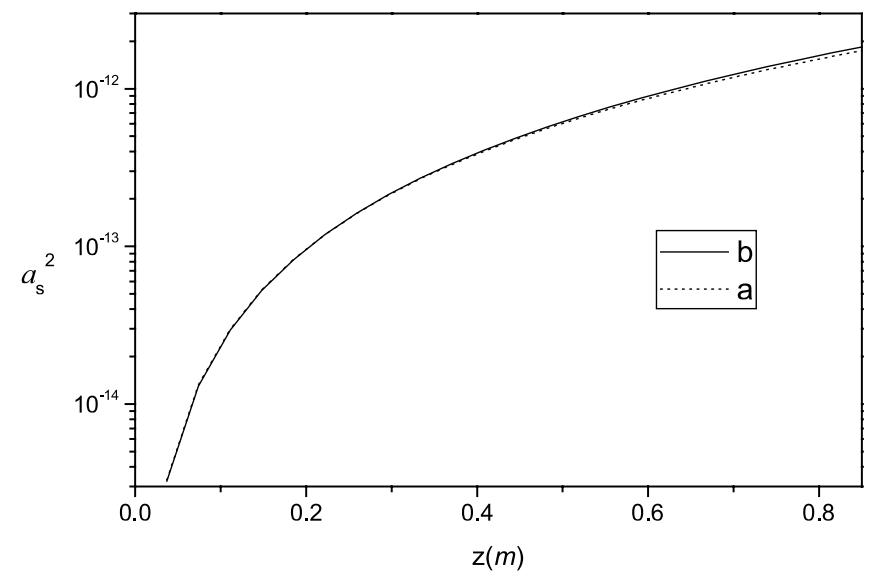

FIG. 2. (a) A result of the analytical formula [Eq. (25)] compared with (b) the result of the linear theory [Eq. (22)] for the CHG. field $a_{s 1}$.

$$
\Delta \gamma_{m} / \gamma<\rho, \quad a_{s 1}<\frac{\left(1+a_{u 1}^{2}\right)}{4 \pi N_{1} a_{u 1} \delta_{p 1}} \rho .
$$

From Eq. (22) the effect of additional energy spread $\Delta \gamma_{m} / \gamma$ on the usual gain process is independent of the harmonic number, and the optimal $\Delta \xi=(n+1) / n$ for the coherent enhancement process does not change much for high harmonic, therefore the optimal seeding laser does not change much with the harmonic number. But as the harmonic number increases, the energy-spread effect factor and the Bessel function term $J_{n}$ decrease; both of them make the gain degradation. The energy-spread factor is more important by comparison. To reduce the energyspread effect we can reduce the dispersive field strength $\left(N_{d}\right)$, but in this case $J_{n}(n \Delta \xi)$ is also decreased. The numerical calculation is needed to get the optimal parameters for a given practical situation.

\section{SUMMARY}

We have analytically derived the optical field evolution equations for both the optical klystron and the harmonic optical klystron FEL configurations. A concise optical field evolution equation is given for the linear regime of OK,. It not only gives small signal gain but also is valid for high gain. The effect of the noninteger dispersive parameter $N_{d}$ is shown explicitly for the first time. The harmonic optical klystron with seeding laser are analyzed for both the CHG mode and the HGHG mode. By numerically solving Eqs. (1) and (16) the harmonic generation problem including the effects of energy spread, energy modulation, and the dispersion in whole process can be easily described. The linear theory is given [Eq. (22)]. It is valid from the initial (CHG mode) to near saturation of the HGHG mode and will be helpful to related theoretical analysis. For the CHG mode the analytical formula is given further, and the advantages of HOK over OK were demonstrated: the radiation intensity can be increased with optimized system parameters, the energy-spread effect will be reduced, and besides the odd harmonic the HOK also can operate at the even harmonic of the seed laser. At last the optimal parameters for harmonic amplifier FEL are discussed briefly.

[1] N. A. Vinokurov, in Proceedings of the 10th International Conference on Particle Accelerators, Serpukhov, 1977, Vol. 2, p. 454.

[2] J. C. Gallardo and C. Pellegrini, Nucl. Instrum. Methods Phys. Res., Sect. A 296, 448 (1990).

[3] R. Bonofacio, et al., Phys. Rev. A 45, 4091 (1992).

[4] R.Coisson and F. De martini, Physics of Quantum Electronics (Addison-Wesley, Reading, MA, 1982), Vol. 9, p. 939.

[5] J.M. Ortega et al., IEEE J. Quantum Electron. 21, 909 (1985). 
[6] Q. K. Jia, High Power Laser Part. Beams 6, 450 (1994) [Nucl. Instrum. Methods Phys. Res., Sect. A 407, 246 (1998)].

[7] L. H. Yu, Phys. Rev. A 44, 5178 (1991).

[8] R. Bonofacio et al., Nucl. Instrum. Methods Phys. Res., Sect. A 296, 787 (1990).

[9] V. N. Litvinenko, Nucl. Instrum. Methods Phys. Res., Sect. A 304, 463 (1991).

[10] G. R. Neil, H. P. Freund, Nucl. Instrum. Methods Phys. Res., Sect. A 475, 381 (2001).

[11] J. Wu, L. H. Yu, Nucl. Instrum. Methods Phys. Res., Sect. A 475, 104 (2001).

[12] E. L. Saldin et al., Opt. Commun. 202, 169 (2002).

[13] W. Brefield et al., Nucl. Instrum. Methods Phys. Res., Sect. A 483, 80 (2002).

[14] P. Elleaume, in Physics of Quantum Electronics, edited by S. Jacobs et al. (Addison-Wesley, Reading, MA, 1982), Vol. 8, pp. 119-151.

[15] W. B. Colson and I. Boscolo, Phys. Rev. A 31, 2353 (1985).
[16] Bartolini et al., Opt. Commun. 235, 395 (2004).

[17] Avner Amir and Yuval Greenzweig, Phys. Rev. A 34, 4809 (1986).

[18] Q. K. Jia, Phys. Lett. A 133, 399 (1988); Appl. Phys. B 49, 541 (1989).

[19] E. L. Saldin, E. Schneidmiller, and M. Yurkov, The Physics of Free-Electron Lasers (Springer-Verlag, Berlin, 1999).

[20] L. H. Yu et al., Nucl. Instrum. Methods Phys. Res., Sect. A 445, 301 (2000).

[21] Q. K. Jia, Nucl. Instrum. Methods Phys. Res., Sect. A 519, 489 (2004).

[22] Q. K. Jia, in Proceedings of the Eighth Particle Accelerator Physics Symposium, Mianyang, 2002, pp. 162-166 (in Chinese).

[23] Li Yuhui, Jia Qika, and Zhang Shancai, in Proceedings of the FEL 2004 Conference (Comitato Conferenze Elettra, Trieste, 2005). 